en prenant $\log (26$ Aurigae $)=0.000$, le nombre de comparaisons et celui d'étoiles avec lesquelles la Nouvelle a été comparée. Les logarithmes de l'intensité représentent le résultat de toutes les observations et sont parfaitement suffisants pour tous les calculs photométriques. Mais comme on veut toujours avoir des magnitudes, j'ai déterminé par une construction graphique le coefficient logarithmique et les magnitudes corrigées des étoiles

$\begin{array}{cc}\chi \text { Aurigae } & 5.07 \\ 26 & 5.72 \\ \text { A } & 7.21 \\ \text { B } & \mathbf{8 . 9 9} \\ \text { Coefficient } \log = & 0.443 .\end{array}$

La dernière colonne donne l'intensité ainsi obtenue, exprimée en magnitudes qui répondent au système de BD.

\begin{tabular}{|c|c|c|c|c|c|}
\hline I 892 & t. m. Mosc. & $\log$ int. & $\mathrm{Cp}_{\mathrm{p}}$ & Et. & mg. \\
\hline Tévr. 9 & $9^{h} 5^{2^{m}}$ & 0.153 & 3 & I & $5 \cdot 37$ \\
\hline 10 & 929 & 0.146 & 2 & 2 & $5 \cdot 39$ \\
\hline I 5 & 90 & 0.064 & 4 & 2 & $5 \cdot 5^{8}$ \\
\hline 7 & 754 & 0.066 & 4 & 2 & 5.57 \\
\hline 20 & 812 & 0.040 & 4 & $\mathbf{I}$ & 5.63 \\
\hline 22 & 747 & $9.92 \mathrm{I}$ & 4 & 3 & 5.90 \\
\hline 24 & 89 & 9.917 & 3 & 2 & $5 \cdot 91$ \\
\hline 27 & 842 & 0.070 & 6 & 3 & $5 \cdot 5^{6}$ \\
\hline Mars 4 & 850 & 0.031 & 3 & 3 & 5.65 \\
\hline I 2 & 852 & 9.088 & 3 & 2 & 7.77 \\
\hline 14 & 725 & $8.9^{8} 3$ & 2 & I & 8.01 \\
\hline I 5 & 715 & 8.876 & 2 & 1 & 8.25 \\
\hline 16 & 825 & 8.766 & 2 & 2 & $8.5^{\circ}$ \\
\hline 17 & 918 & $8.62 \mathrm{I}$ & 2 & 2 & 8.83 \\
\hline 30 & 925 & 7.133 & 2 & $\mathbf{I}$ & \\
\hline
\end{tabular}
mauvaises.

Févr. 9. Les conditions atmosphériques étaient très

Mars 30. La Nouvelle a été comparée avec une petite étoile de ro $\mathrm{mg}$.

Les observations seront publiées en détail dans la. prochaine livraison de nos Annnales, qui est sous presse.

Moscou, le $18 / 3^{\circ}$ Juin 1892 .

\section{\$2.}

Les nombreuses mesures photométriques de l'étoile nouvelle, publiées dans les journaux, sont précisément en rapport avec le développement considérable que l'astrophotométrie a pris le dernier temps. Néanmoins, les observations à l'œil, d'après la méthode d'Argelander, auront toujours une grande valeur, mais il faut $y$ apporter quelques améliorations.

D'abord, les étoiles de comparaison doivent être déterminées photométriquement, ce qui peut être fait plus tard par quelque autre observateur, comme on procède avec les coordonnées des étoiles de comparaison en cas de comètes.

Puis, dans cette sorte d'observations il y a une forte équation personnelle, qui dépend de la position relative des étoiles à comparer, et qui peut de beaucoup surpasser l'erreur de comparaison. Il me semble que, at moins pour les étoiles télescopiques, on peut éliminer cette équation au moyen d'un oculaire spécial.

Je profite de cette occasion pour diriger l'attention de MM. les opticiens sur la construction d'un parail oculaire.

Il doit se composer de deux oculaires réunis de manière à ce qu'on puisse les substituer l'un à l'autre, momentanément et avec la plus grande facilité. Un des oculaires est astronomique, ordinaire, c'est à dire renversant les images, l'autre - terrestre. Les deux se composent d'un même nombre de lentilles et ont le même champ de la vision et le même grossissement. S'ils ne peuvent pas être parfaitement achromatiques, il faut répartir le défaut entre les deux.

Comme les étoiles à comparer doivent être dans le même champ de la vision, il convient d'avoir un faible grossissement et le champ de la vision le plus grand possible; c'est l'expérience qui montrera quelle dimension de lunette convient le mieux aux étoiles variables connues à présent. Quant au changement de la position relative des étoiles par un prisme oculaire ordinaire il faut renoncer à son usage.

Prof. W. Ceraski.

\title{
Osservazioni della cometa periodica di Winnecke $(1892 \ldots)$
}

fatte all' equatoriale di $25^{\mathrm{cm}}$ di a pertura del R. Osservatorio del Collegio Romano da E. Millosevich.

Le seguenti osservazioni sono fatte al micrometro filare con forte ingrandimento e a fili lucidi; $l$ ' astro rimase relativamente poco appariscente con nucleo piccolo di $10^{\mathrm{ma}}$ grandezza e nebulosità dissimetrica. Dopo il 29 giugno la posizione della cometa, avvicinantesi il perielio, non permise osservazioni esatte. I confronti coll' effemeride rigorosa. di Haerdtl (A. N. 3083) vennero fatti con cura, interpolando i luoghi dell' effemeride con differenze quarte.

\begin{tabular}{|c|c|c|c|c|c|c|c|c|c|c|}
\hline 1892 & t. m. Roma & $\Delta \alpha$ & $\Delta \delta$ & Cf. & $\alpha$ app. & $\log p \cdot \Delta$ & $\delta$ app. & $\log p \cdot \Delta$ & Red. ad 1. app. & * \\
\hline iug. 17 & $10^{\mathrm{h}} 34^{\mathrm{m}} 28^{\mathrm{s}}$ & $+2^{m} 34^{\mathrm{s}} 6 \mathrm{I}$ & - I $5 I^{n}: 1$ & $9 \cdot 3$ & $10^{\mathrm{h}} 12^{\mathrm{m}} 32^{\mathrm{s}} .84$ & 9.7624 & $+40^{\circ} 33^{\prime}$ I $3^{\prime \prime}$. & $0.664 \mathrm{I}$ & $+0.35+10.4$ & $\mathbf{I}$ \\
\hline 20 & 9106 & +21.08 & +228.8 & 12.4 & $958 \quad 17.60$ & 9.7459 & $\begin{array}{lll}+3936 & 0.7\end{array}$ & $0.55^{8} 5$ & $+0.23+10.2$ & 2 \\
\hline 22 & 9376 & $-3 \quad 8.13$ & $+85^{0.1}$ & I 2.4 & $9 \quad 46 \quad 18.44$ & 9.7508 & +384434.9 & 0.6504 & $+0.16+9.9$ & 3 \\
\hline 23 & $935 \quad 35$ & -I 46.64 & +819.5 & 6.2 & 93937.56 & 9.7480 & +38 I4 11.7 & $0.674 I$ & $+0.11+9.7$ & 4 \\
\hline 25 & 94053 & -0 32.85 & +610.1 & 6.4 & 2432.10 & $9 \cdot 73^{6 I}$ & $+37 \circ 33.7$ & 0.7226 & $+0.0 x+9.3$ & 5 \\
\hline 26 & 9148 & - 17.19 & 47.1 & 11.4 & $916 \quad 16.27$ & $9.735^{2}$ & +36 1 $7 \quad 4.7$ & $0.705 \mathrm{I}$ & $-0.06+9.1$ & 6 \\
\hline 28 & 92224 & +359.23 & 27.9 & 6.2 & $\begin{array}{lll}8 & 57 & 29.51\end{array}$ & $9.7 \times 47$ & +342846.2 & 0.7578 & $-0.15+8.5$ & 7 \\
\hline 29 & 91443 & +o 4.39 & -4 & 16.2 & $\begin{array}{lll}847 & 15.41\end{array}$ & 9.7060 & +332352.4 & 0.7681 & $-0.18+8.2$ & 8 \\
\hline
\end{tabular}


Luoghi medii delle stelle di confronto r892.0.

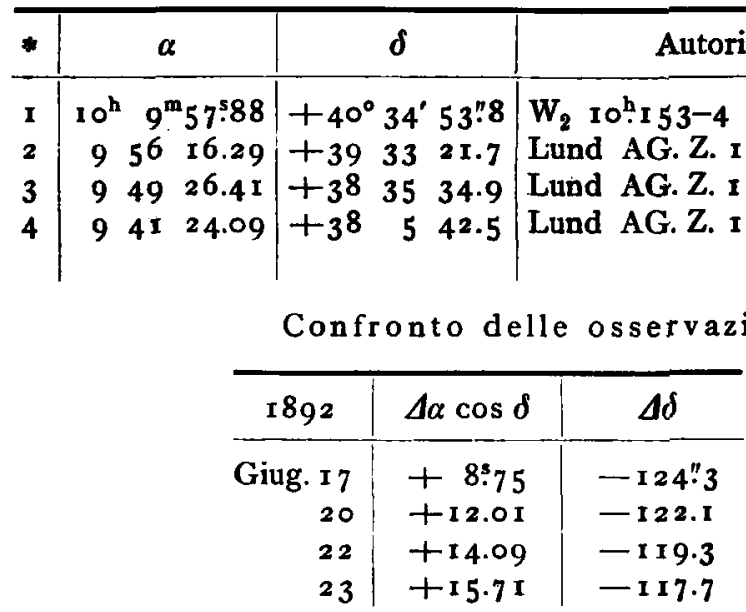

Roma 1892 Luglio 3 .

\begin{tabular}{|c|c|c|c|}
\hline * & $\alpha$ & $\boldsymbol{d}$ & Autorità \\
\hline 5 & $9^{h} 25^{m} \quad 4^{s} \cdot 94$ & $+36^{\circ} 54^{\circ} \times 4^{\prime \prime} 3$ & $\begin{array}{l}\text { Lund AG.Z. I7I, I } 75 \text {. } \\
\text { Rossa di } 7^{\mathrm{m}} \text {. }\end{array}$ \\
\hline 6 & 9 I $723.5^{2}$ & $+36 \times 28.5$ & BB.VI +36:1952 \\
\hline $\begin{array}{l}7 \\
8\end{array}$ & 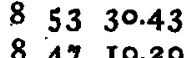 & +34219.8 & Leid. AG. Z. $32,34,283$ \\
\hline
\end{tabular}

\section{Elementi ellittici di (303) Josephina}

in base alla prima e seconda opposizione.

Epoca 1891 Febbrajo $2012^{\text {h }}$ Berlino.

$$
\begin{aligned}
& M=79^{\circ} 49^{\prime} 54^{\prime \prime} 9 \\
& \infty=73 \text { 13 53.3 } \\
& \Omega=345 \quad 16 \quad 17.6 \quad 1892.0 \\
& i=65427.6 \text { ) } \\
& \varphi=33649.4 \\
& \mu=642 " 61925 \\
& \log a=0.4947018
\end{aligned}
$$

Questi elementi (omesse le perturbazioni) rappresentano le osservazioni nel modo seguente $(\mathrm{O}-\mathrm{C})$ :

$$
\begin{array}{rrrr}
1891 & \text { Febbr. } 14.5 & +0.01 & -0.8 \\
\text { 28.5 } & -0.32 & -5.4 \\
\text { Marzo r 3.5 } & -0.47 & -5.6 \\
\text { Aprile 1.5 } & +0.03 & -3.5 \\
\text { Maggio r 2.0 } & +0.07 & -1.4 \\
1892 & \text { Maggio 15.5 } & +0.02 & 0.0
\end{array}
$$

In seconda opposizione $\mathrm{l}$ ' astro fu osservato da Charlois a Nizza.

Roma 1892 Luglio 5 .

E. Millosevich.

\section{Ueber die Nova Aurigae.}

Das Wolsingham Observatory Circular Nr. 33 lautet:

$\triangleright$ Mr. H. Corder having informed me that the Nova Aurigae has increased, it was examined here Aug. 2 I and found to be $9 \% 2$, spectrum monochromatic. One intense line (500?).

T. E. Espin.к

Sofort nach Eintreffen des Circulars, Aug. 24, wurden einige wenige Sternwarten telegraphisch in Kenntniss gesetzt und um Verificirung der Beobachtung gebeten. Die letztere hat sich durch das andauernd schlechte Wetter leider einige Tage verzögert; erst am 2. Sept. Vormittags ging folgende Mittheilung von Prof. Küstner bei der Centralstelle ein :

„Endlich hat das Wetter eine Beobachtung der Nova gestattet. Ich schätze : Aug. 3 I $_{1} \mathbf{I}_{\mathbf{4}} \mathbf{m}$ M. Z. Bonn Nova $3 \frac{1}{2}$ Stufen heller als der nördlich folgende Stern ro. Grösse (Krueger's Stern von $185^{8}$ ), bei klarer Luft aber tiefem Stand. Die Nova ist demnach heute wieder so hell wie März 21 .\&

$$
\text { Bonn } 1892 \text { August } 3 \mathbf{I} \text {. F. Küstner. }
$$

Diese Beobachtung wurde gemeinsam mit der Mittheilung von Espin am 2. Sept. den Mitgliedern der Centralstelle sowie den amerikanischen Sternwarten telegraphisch übermittelt.

Telegramm aus Greenwich, eingegangen Sept. 2 Abends:

\Nova Aurigae photographed here August 30 about twelfth magnitude. Christie.k 